\title{
Electromagnetic Arbitrary Waveform Generation with Broadband Incoherent Light Sources
}

\author{
V. Torres-Company, J. Lancis, P. Andrés and L.R. Chen
}

$\mathrm{n}$ recent years, advances in pulse-

shaping technology have shown great potential for applications in microwave photonics. ${ }^{1,2}$ Researchers' interest is partly motivated by the fact that the generation of arbitrary electromagnetic signals with 1-50 GHz frequency content is a challenge for purely electronic systems. Broad bandwidth signals could have a positive impact on high-speed wireless communication systems and find interesting applications in radar, remote sensing and electronic-equipment test measurements. ${ }^{1}$

Previously demonstrated photonicbased arbitrary waveform generators (AWGs) fall easily within the desired frequency range. Their principle of operation is based on the following general scheme. First, a broadband coherent signal (e.g., from a mode-locked laser) is synthesized in a user-defined way in the optical domain. Usually, pulse shapers based on spatial light modulators are preferred to all-fiber configurations because they provide reconfiguration capabilities. $^{2}$ Once the synthesis is performed, the light intensity is transferred to the electrical domain simply by using a highspeed photodetector.

In this way, the detector sets the upper limit on the achievable electrical bandwidth. Therefore, while obtaining highfrequency electrical signals is a relatively straightforward task with mode-locked lasers, reaching the low-frequency regime remains a challenge.

In 2003, this problem was circumvented thanks to the coherent "wavelength-to-time mapping technique" demonstrated by Jalali's group at UCLA. ${ }^{3}$ This scheme consists of synthesizing the energy spectrum of a coherent broadband signal with a Fourier-transform pulse shaper and later transferring the designed spectral shape into the electrical domain by stretching the optical pulse

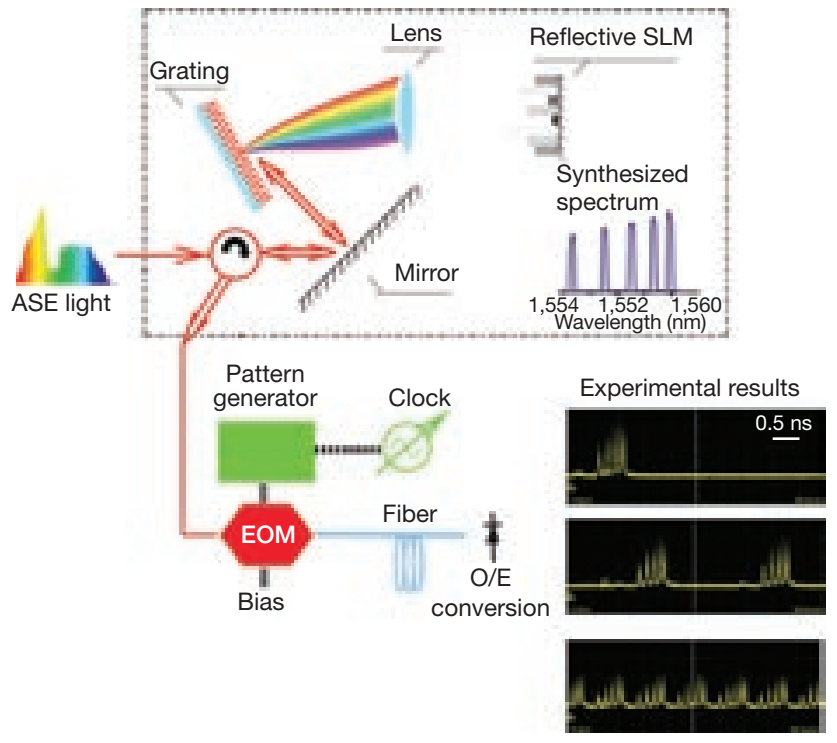

Example of generating a chirped sinusoidal signal: The incoherent radiation is spectrally shaped with a Fourier transform pulse shaper. Once the spectrum is synthesized, the radiation is modulated with an external modulator. Finally, the light is stretched in a fiber long enough so that the output-averaged intensity becomes a scaled replica of the synthesized energy spectrum. The scaling factor is exactly the same as in the coherent version and, therefore, the previous advantageous features are preserved.

in a dispersive medium (e.g., fiber) and subsequently detecting it. Accordingly, the scaling factor of the resulting electromagnetic waveform can be tunable by adjusting the amount of dispersion.

We have gone one step further and shown that this widely used system can be operated with a spectrally incoherent light source such as amplified spontaneous emission (ASE). The physical mechanism behind this configuration relies on the temporal version of the vanCittertZernike theorem formulated by Dorrer in $2004 .{ }^{4}$ This theorem extends the previous wavelength-to-time mapping to the incoherent regime. In 2006, we suggested a theory of how this could be used for AWG. This year, we achieved the first experimental results. ${ }^{5}$

The setup is shown in the figure. The main goal is to avoid the use of a mode-locked laser. Apart from being an interesting economic alternative, this allows us to control the repetition rate of the electromagnetic waveform with an external clock. This is a key feature to perform continuously operating radiofrequency waveforms. To date, we have generated arbitrary electrical signals with frequency content around $1-10 \mathrm{GHz}$ using standard $10 \mathrm{~Gb} / \mathrm{s}$ telecommunications equipment. $\triangle$

V. Torres-Company (vtorres@fca.uji.es) and J. Lancis are with the departament de fisica, Universitat Jaume I, in Castello, Spain. P. Andrés is with the departamento de Optica, Universitat Valencia, in Burjassot, Spain. L.R. Chen is with the department of electrical and computer engineering at McGill University in Montreal, Canada.

References

1. J. Capmany and D. Novak. Nature Photon. 1, 319 (2007).

2. J.D. McKinney et al. Opt. Photon. News 17, 24 (2006).

3. J. Chou et al. IEEE Photon. Technol. Lett. 15, 581 (2003)

4. C. Dorrer. J. Opt. Soc. Am. B 21, 1417 (2004)

5. V. Torres-Company et al. J. Lightwave Technol. 26, 2476 (2008). 